\title{
AVALIAÇÃO DO EQUILÍBRIO ESTÁTICO EM INDIVÍDUOS AMPUTADOS DE MEMBROS INFERIORES ATRAVÉS DA BIOFOTOGRAMETRIA COMPUTADORIZADA
}

\author{
Baraúna MA ${ }^{1}$, Duarte $\mathrm{F}^{2}$, Sanchez HM ${ }^{1}$, Canto RST $^{1}$, Malusá S $^{3}$, Campelo-Silva CD ${ }^{2}$, \\ VENTURA-SiLVA RA ${ }^{4}$ \\ ${ }^{1}$ PPG - Fisioterapia, Centro Universitário do Triângulo, Uberlândia, MG \\ ${ }^{2}$ Departamento de Fisioterapia, Universidade Luterana do Brasil , Porto Alegre, RS \\ ${ }^{3}$ Departamento de Pedagogia, Universidade Federal de Uberlândia, Uberlândia, MG \\ ${ }^{4}$ Departamento de Fisioterapia, Universidade de Gurupi, Gurupi, TO \\ Correspondência para: Mário Antônio Baraúna, Centro Universitário do Triângulo - UNITRI, Av. Nicomedes Alves \\ dos Santos, nº 4545, Bairro Gávea, CEP 38411-106, Uberlândia, MG, e-mail: barauna@unitri.edu.br
}

Recebido: 24/02/2005 - Aceito: 22/09/2005

\begin{abstract}
RESUMO
Contextualização: Para que sejam realizadas atividades funcionais da vida diária é primordial a manutenção do equilíbrio estático, possibilitando posturas, movimentos e respostas adequadas. Objetivos: O presente trabalho teve como finalidade avaliar e comparar, através do teste de Romberg adaptado, as oscilações do equilíbrio estático de amputados protetizados de membros inferiores com indivíduos não amputados. Método: Foram avaliados 33 indivíduos do sexo masculino com idade média de 33,38 anos, divididos em três diferentes grupos. O grupo A foi composto por 12 indivíduos amputados que utilizavam prótese transfemural. O grupo B foi formado por 9 indivíduos amputados transtibiais. O grupo controle C continha 12 indivíduos não amputados. Cada indivíduo foi filmado nos planos frontal e sagital, avaliado quadro a quadro para a seleção de momentos de maiores oscilações. As imagens selecionadas foram quantificadas através da Biofotogrametria Computadorizada, utilizandose o programa ALCimage 2.1 ${ }^{\circledR}$. Resultados: Os resultados mostraram diferenças significativas entre as oscilações anteriores para o grupo A (transfemural) e entre as oscilações para o lado esquerdo, no grupo B. Conclusão: A partir destes resultados, concluise que amputados de membros inferiores apresentam uma tendência de oscilação mais significante em direção anterior e para o lado contra-lateral à prótese. Com o avanço da idade, nota-se uma menor tendência à oscilação posterior.
\end{abstract}

Palavras-chave: amputação, equilíbrio estático, biofotogrametria computadorizada.

\section{ABSTRACT \\ Evaluation of the Static Balance Lower Limb Amputeers by Means of Computerized Biophotogrammetry}

Background: To accomplish day-to-day functional activities, it is fundamentally important to maintain static balance, thereby enabling appropriate postures, movements and responses. Objective: The purpose of this study was to evaluate oscillations in static balance among amputees with lower limb prostheses, by means of an adapted Romberg test, and to compare such oscillations with those among individuals without amputations. Method: Thirty-three male individuals of mean age 33.38 years were evaluated, in three different groups. Group A was composed of 12 amputees who were utilizing a transfemoral prosthesis. Group B was formed by 9 individuals with a transtibial amputation. Group C (control) contained 12 normal individuals. All subjects were filmed in the frontal and sagittal planes, and their movements were evaluated frame by frame to select the moments of greatest oscillation. The images selected were quantified by means of computerized biophotogrammetry using the program ALCimage $2.1^{\circledR}$. Results: The results showed significant differences among the anterior oscillations for the group A and among the left-side oscillations for group B. Conclusion: From these results, it was concluded that lower-limb amputees present a tendency towards more significant oscillations in the anterior direction and contralaterally to the prosthesis. With advancing age, a lesser tendency towards posterior oscillation was noted.

Key-Words: amputation, static balance, computerized biophotogrammetry. 


\section{INTRODUÇÃO}

Existem vários conceitos de amputação. Boccolini ${ }^{1} \mathrm{e}$ Carvalho $^{2}$ a definem como sendo a retirada cirúrgica, total ou parcial, de um membro. Estes autores afirmam ainda que as amputações possam ter indicações eletivas, como nos casos das doenças e más-formações ou indicações de urgência, como em traumas importantes e infecções graves.

Após a amputação a utilização de uma prótese oferece uma imagem corporal normal, ajudando o indivíduo a desenvolver maior confiança e habilidades físicas e melhorando a sua qualidade de vida indivíduo ${ }^{3,4}$.

Boccolini ${ }^{1}$, Lusardi e Nielsen ${ }^{5}$ e Carvalho ${ }^{2}$ afirmam que a amputação transtibial é realizada entre a articulação tibiotársica e a do joelho, sendo esta amputação no mínimo duas vezes mais comum que em outros níveis. Já a amputação transfemural, segundo Calmel et al. ${ }^{6}$ e Carvalho ${ }^{2}$ refere-se a toda amputação realizada entre a articulação do joelho e quadril.

Para que haja a manutenção do equilíbrio, faz-se necessário que estejam íntegros os elementos anatômicos e funcionais, que compreendem o aparelho vestibular, a visão, os centros nervosos, o sistema proprioceptivo e o sistema musculoesquelético ${ }^{7,8,9,10,11,12,13}$. Calmel et al. ${ }^{6}$ têm mostrado que o equilíbrio está alterado em amputados de membros inferiores. Isakov et al. ${ }^{14}$, Mouchnino ${ }^{15}$ e Buckley et al. ${ }^{16}$ encontraram aumento da oscilação em amputados de membros inferiores quando comparados ao grupo controle.

Sabe-se que o indivíduo amputado de membro inferior pode apresentar dificuldades na manutenção do equilíbrio estático, o que pode gerar quedas, as quais em casos mais graves, podem levar a fraturas ${ }^{17,18}$. Baraúna et al. ${ }^{13}$ acreditam que a avaliação do equilíbrio estático possa ser um suporte prognóstico para o desenvolvimento de esquemas preventivos e propostas terapêuticas, evitando assim, complicações decorrentes do desequilíbrio.

A eleição do teste de Romberg adaptado para avaliação do equilíbrio estático, em 30 segundos é justificada pelos resultados obtidos por Presumido et al. ${ }^{10} \mathrm{e}$ Baraúna ${ }^{17}$, os quais demonstraram que as maiores oscilações ocorrem no intervalo de 30 segundos.

A Biofotogrametria Computadorizada desenvolveuse pela aplicação dos princípios fotogramétricos às imagens fotográficas obtidas em movimentos corporais. A essas imagens foram aplicadas bases de fotointerpretação, gerandose uma nova ferramenta de estudo da cinemática ${ }^{19}$. Assim, a Biofotogrametria Computadorizada é um recurso que pode ser usado na avaliação, para diagnóstico físico funcional pelos fisioterapeutas, em diferentes áreas, sendo que esta já foi utilizada em vários estudos, nos quais foram demonstradas a sua validade ${ }^{20}$.
Portanto, os objetivos deste estudo foram verificar através da Biofotogrametria Computadorizada o equilíbrio estático de indivíduos amputados transfemurais e transtibiais, comparando-os a indivíduos não amputados e verificar se ocorrem alterações do equilíbrio, dentre os diferentes níveis de amputações de membros inferiores.

\section{MÉTODO}

O presente estudo avaliou 33 indivíduos do sexo masculino (com idade média de 33,38 $\pm 2,02$ ) divididos em três diferentes grupos. O grupo A foi composto por 12 indivíduos amputados que utilizavam prótese transfemural. O grupo B foi formado por 9 indivíduos amputados transtibiais, e o grupo $C$ que continha 12 indivíduos não amputados.

Os indivíduos amputados foram encaminhados pela Associação Mineira de Paraplegia e Ortosolutions - Próteses e Órteses Ltda, ambos situados em Belo Horizonte, MG. Era imprescindível que no encaminhamento constasse o tempo de protetização, idade e protocolo de verificação protética.

O local eleito para recolha de dados foi a Ortosolutions - Próteses e Órteses Ltda, onde estavam instalados todos os instrumentos utilizados para avaliação, era devidamente nivelado, com boa iluminação e dimensões adequadas. A análise dos dados foi realizada no Laboratório de Análise do Movimento do UNITRI - Centro Universitário do Triângulo.

Antes da recolha dos dados, o estudo foi submetido ao comitê de Ética em Pesquisas em Humanos do UNITRI (Centro Universitário do Triângulo), do qual recebeu um parecer de concordância para realização da pesquisa. Foi feita a leitura do termo de consentimento informado e os indivíduos assinaram o mesmo concordando em participar do estudo.

Para que a amostragem apresentasse características semelhantes para altura, peso e idade adotaram-se critérios de admissão para os três grupos. Deste modo, incluíramse neste estudo os indivíduos amputados bem adaptados ao aparelho protético que não apresentavam idade inferior a 18 anos ou superior a 65 anos de idade, amputados por trauma; indivíduos que utilizavam aparelhos protéticos entre 1,5 e 5 anos e indivíduos que não possuíssem alterações musculoesqueléticas que os impossibilitassem de permanecer em ortostatismo. No grupo controle, foram incluídos indivíduos sem patologias ou dor recente nos membros inferiores, bem como aqueles que possuíssem doenças musculoesqueléticas as quais comprometessem o seu equilíbrio. Como critérios de exclusão, observaram-se a discrepância dos membros inferiores, pois segundo Ramos e Demyer ${ }^{7}$ grandes diferenças podem acarretar o desequilíbrio e hipertensos, pois estes podem ter desequilíbrios causados pela alta pressão sangüínea. 
Após a inclusão do voluntário, foi preenchida a ficha de avaliação. Todos os participantes eram pesados em uma balança devidamente aferida pelo INMETRO (Instituto Nacional de Metrologia, Normalização e Qualidade Industrial). Tendo sido concluídas essas etapas, iniciava-se a parte de realização do teste de equilíbrio para coleta de dados. De início, um capacete com ponteira, pesando aproximadamente $20 \mathrm{~g}$ foi posicionado com sua ponteira em alinhamento com a glabela, a fim de se avaliarem as oscilações no plano frontal. Para quantificação das oscilações do plano sagital, a referência utilizada foi o pavilhão auditivo direito em todos os indivíduos. A câmera filmadora foi posicionada em nível e em prumo, a uma distância de 3,60 metros e a uma altura de 1,40 metros do solo; sendo assim mantidas para a filmagem de todos os indivíduos. As imagens coletadas foram do perfil direito e em apresentação anterior.

Para verificar as oscilações do corpo em equilíbrio estático, foi utilizado um protocolo de estudo que empregou a Biofotogrametria Computadorizada como instrumento quantificador angular do desvio da linha de equilíbrio, sendo aplicado o teste de Romberg adaptado ${ }^{17}$. Com o capacete alinhado ao fio de prumo, os indivíduos adotavam posição ortostática por 30 segundos, permanecendo com os olhos fechados e pés paralelos. A avaliação do equilíbrio foi mensurada através da observação das oscilações posteriores, anteriores e látero-laterais, calculadas em graus.

A fim de se obter o momento de maior oscilação anterior (MOA), posterior (MOP), lateral para a direita (MOD) e para esquerda (MOE), as imagens eram analisadas quadro a quadro, e, no instante de maior oscilação a imagem era gravada no computador. Este processo foi repetido para maior precisão e confiabilidade do exato momento de maior oscilação ${ }^{13}$.

As imagens selecionadas foram então analisadas pela Biofotogrametria Computadorizada, no programa Alcimage $2.1^{\circledR}$, determinando-se os valores angulares de maneira precisa e fidedigna ${ }^{19,20}$.

Para o cálculo dos ângulos referentes às oscilações anteriores e posteriores da amostragem, foram demarcados vértices, formando-se triângulos ${ }^{19,20}$. Constituíam-se os vértices: vértice $A$, na ponta da antena; vértice $B$, no fio de prumo ao nível do pavilhão auditivo, e vértice $C$ também no fio de prumo só que ao nível do vértice A (Figuras 1 e 2).

Através dessas demarcações de vértices e formação do triângulo, foram analisados os ângulos de oscilações anteriores e/ou posteriores, formados no vértice $B$, onde foi evidenciado o distanciamento da antena em relação ao fio de prumo, podendo, assim, quantificarem-se os ângulos ${ }^{13,19,20}$ (Figuras 1 e 2 ).

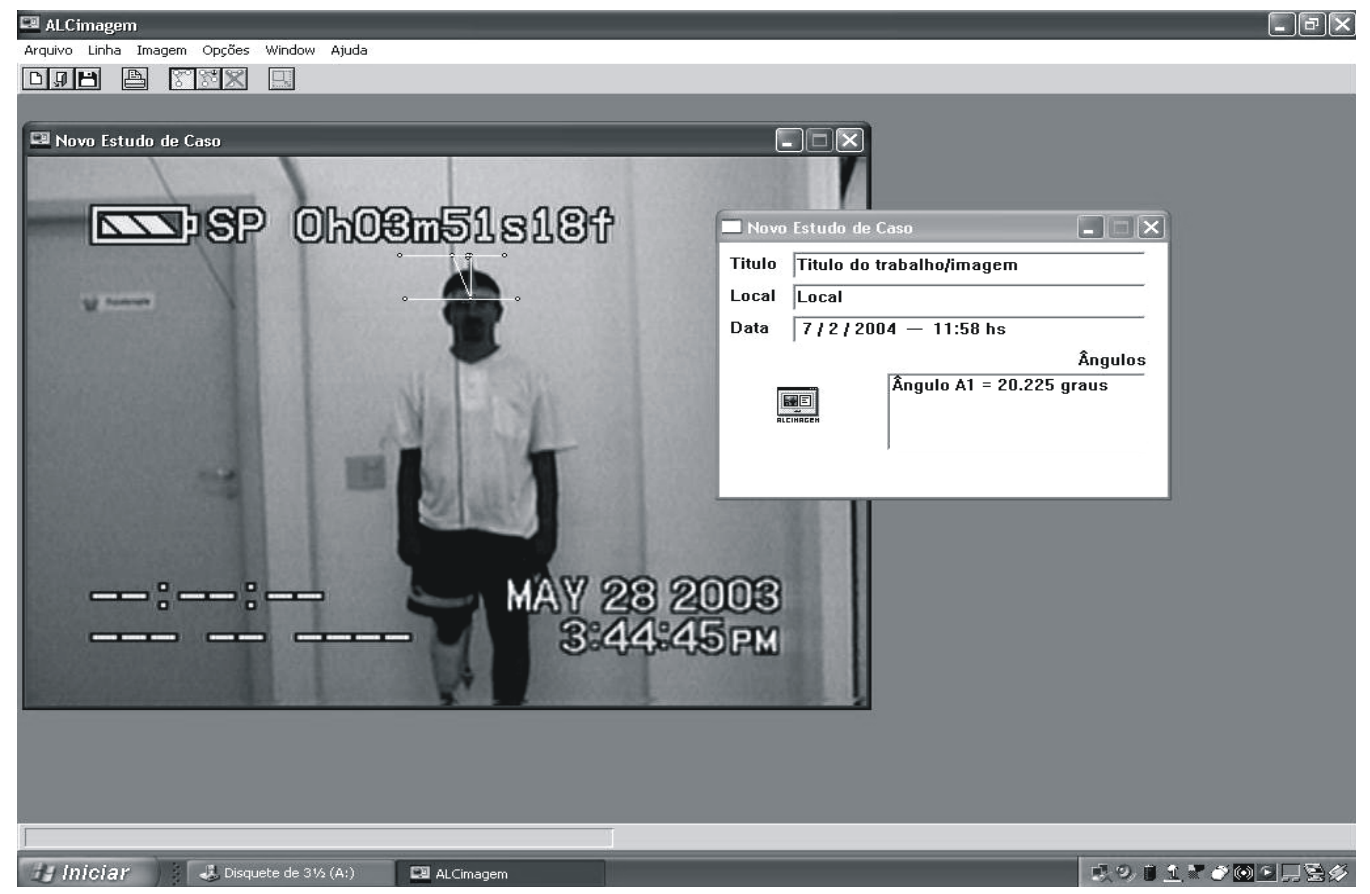

Figura 1. Quantificação angular de oscilação no plano frontal (látero-lateral) utilizando o Programa ALCimage $2.1^{\circledR}$. Demarcação dos pontos para formação do ângulo. 


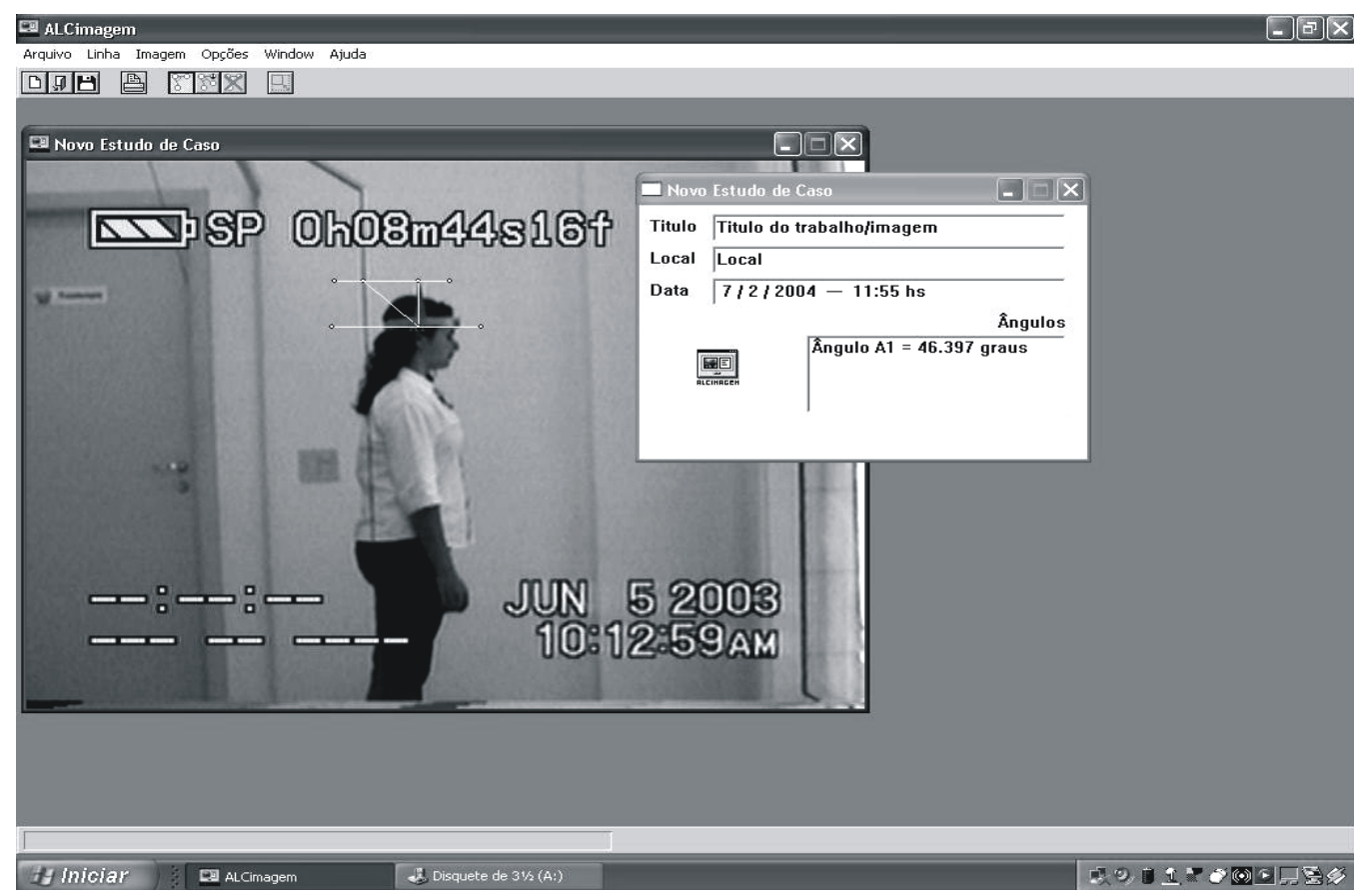

Figura 2. Quantificação angular de oscilação no plano sagital (anteroposterior) utilizando o Programa ALCimage $2.1^{\circledR}$ Demarcação dos pontos para formação do ângulo.

É importante ressaltar que os indivíduos do grupo A e B permaneceram com os calçados originais do alinhamento da prótese durante todo o tempo da realização do teste, não sofrendo, assim, alterações decorrentes do desnível do alinhamento. Já os indivíduos do grupo C, permaneceram descalços durante todo o tempo da tomada das imagens.

Após terem-se sido estabelecidos os ângulos referentes às oscilações nos planos sagital e frontal, procedeu-se o tratamento estatístico a fim de verificar a ocorrência ou não de significância entre os ângulos. Para tanto, o teste $U$ de Mann-Whitney foi utilizado com a finalidade de comparar as medidas de maior oscilação para ambos os lados, o peso da prótese e o tempo de uso da prótese entre amputados transtibiais e amputados transfemurais, amputados transtibiais e grupo controle, e amputados transfemurais e grupo controle. O teste de Wilcoxon foi aplicado para verificar a ocorrência ou não de significância entre os resultados obtidos dentro de um mesmo grupo. E com o objetivo de verificar a existência ou não de correlações significantes entre as medidas de maior oscilação e as medidas de tempo de uso da prótese, peso da prótese e idade, foi aplicado o Coeficiente de Correlação por Postos de Spearman aos indivíduos do grupo de amputados transtibiais e grupo controle. O nível de significância foi estabelecido em $5 \%$ para ambos os testes.

\section{RESULTADOS}

De acordo com a Tabela 1, os amputados transtibiais, quando comparados com os transfemurais, mostraram diferenças significativamente maiores nas medidas de oscilações à esquerda e tempo de uso da prótese dos itens analisados, sendo que 8 dos 9 indivíduos eram amputados do membro inferior direito.

Os amputados transtibiais comparados com o grupo controle tiveram diferenças significantes nas medidas de maior oscilação à esquerda (Tabela 2).

Na Tabela 3, observam-se diferenças nas medias de maior oscilação anterior entre o grupo dos amputados transfemurais e o grupo não amputado.

As comparações entre as maiores oscilações dentro de um mesmo grupo são demonstradas na Tabela 4, na qual se observam diferenças entre maior oscilação anterior e maior oscilação à direita, nos amputados transtibiais, enquanto que no grupo dos amputados transfemurais ocorreu diferença nas medidas de maior oscilação anterior e maior oscilação à direita, maior oscilação anterior e maior oscilação à esquerda e maior oscilação anterior e maior oscilação posterior. 
Tabela 1. Probabilidades encontradas, quando da aplicação do teste U de Mann-Whitney às medidas obtidas com amputados transtibiais e transfemurais.

\begin{tabular}{lccc}
\hline \multicolumn{1}{c}{ Variáveis analisadas } & Transtibiais & Transfemurais & Valores de p \\
\hline MOA & $18,53 \pm 14,99$ & $14,81 \pm 8,03$ & 1 \\
MOD & $6,01 \pm 9,80$ & $6,25 \pm 5,28$ & 0,2773 \\
MOE & $10,95 \pm 8,66$ & $27,81 \pm 5,09$ & $0,0118^{*}$ \\
MOP & $8,33 \pm 12,54$ & $28,45 \pm 6,94$ & 0,2538 \\
Plano anteroposterior & $26,86 \pm 2,33$ & $17,65 \pm 4,55$ & 0,2806 \\
Plano látero-lateral & $16 \pm 13,68$ & $9,04 \pm 5,75$ & 0,4545 \\
Tempo de uso da prótese & $3,38 \pm 0,1$ & $2,57 \pm 1,14$ & $0,0001^{*}$ \\
Peso da prótese & $1,96 \pm 1,41$ & $3,46 \pm 0,49$ & 0,7759 \\
\hline
\end{tabular}

(*) p<0,05; MOA - maior oscilação anterior, MOD - maior oscilação a direita, MOE - maior oscilação a esquerda, MOP - maior oscilação posterior.

Tabela 2. Probabilidades encontradas, quando da aplicação do teste U de Mann-Whitney às medidas obtidas com amputados transtibiais e com indivíduos do grupo controle.

\begin{tabular}{lccc}
\hline Variáveis analisadas & Transtibiais & Controle & Valores de p \\
\hline MOA & $18,53 \pm 14,99$ & $10,11 \pm 4,63$ & 0,4767 \\
MOD & $6,01 \pm 9,80$ & $3,3 \pm 3,72$ & 0,9387 \\
MOE & $10,95 \pm 8,66$ & $4,09 \pm 4,77$ & $0,0427^{*}$ \\
MOP & $8,33 \pm 12,54$ & $5,64 \pm 5,66$ & 0,8179 \\
Plano anteroposterior & $26,86 \pm 2,33$ & $15,74 \pm 5,10$ & 0,5438 \\
Plano látero-lateral & $16 \pm 13,68$ & $7,42 \pm 2,63$ & 0,5692 \\
\hline
\end{tabular}

$\left(^{*}\right) \mathrm{p}<0,05$; MOA - maior oscilação anterior, MOD - maior oscilação a direita, MOE - maior oscilação a esquerda, MOP - maior oscilação posterior.

Tabela 3. Probabilidades encontradas, quando da aplicação do teste U de Mann-Whitney às medidas obtidas com amputados transfemurais e com indivíduos do grupo controle.

\begin{tabular}{cccc}
\hline Variáveis analisadas & Transfemurais & Controle & Valores de p \\
\hline MOA & $14,81 \pm 8,03$ & $10,11 \pm 4,63$ & $0,0240 *$ \\
MOD & $6,25 \pm 5,28$ & $3,3 \pm 3,72$ & 0,0557 \\
MOE & $27,81 \pm 5,09$ & $4,09 \pm 4,77$ & 0,4257 \\
MOP & $28,45 \pm 6,94$ & $5,64 \pm 5,66$ & 0,1111 \\
Plano anteroposterior & $17,65 \pm 4,55$ & $15,74 \pm 5,10$ & 1,000 \\
Plano látero-lateral & $9,04 \pm 5,75$ & $7,42 \pm 2,63$ & 0,3547
\end{tabular}

$\overline{(*)}$ p<0,05; MOA - maior oscilação anterior, MOD - maior oscilação a direita, MOE - maior oscilação a esquerda, MOP - maior oscilação posterior. 
Tabela 4. Probabilidades encontradas, quando da aplicação do teste de Wilcoxon às medidas obtidas com amputados transtibiais e transfemurais.

\begin{tabular}{|c|c|c|}
\hline Variáveis analisadas & MA e DP da $1^{\mathrm{a}}$ e $2^{\mathrm{a}}$ variáveis & Valores de p \\
\hline Transtibial & & Transtibial \\
\hline MOA X MOD & $18,53 \pm 14,99 \times 6,01 \pm 9,80$ & $0,0077^{*}$ \\
\hline MOA X MOE & $18,53 \pm 14,99 \times 10,95 \pm 8,66$ & 0,0663 \\
\hline MOA X MOP & $18,53 \pm 14,99 \times 8,33 \pm 12,54$ & 0,0663 \\
\hline MOD X MOE & $6,01 \pm 9,80 \times 10,95 \pm 8,66$ & 0,1097 \\
\hline MOD X MOP & $6,01 \pm 9,80 \times 8,33 \pm 12,54$ & 0,4652 \\
\hline MOE X MOP & $10,95 \pm 8,66 \times 8,33 \pm 12,54$ & 0,5754 \\
\hline Transfemural & & Transfemural \\
\hline MOA X MOD & $14,81 \pm 8,03$ X 6,25 $\pm 5,28$ & $0,0229 *$ \\
\hline MOA X MOE & 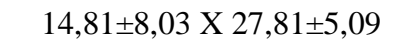 & $0,0051^{*}$ \\
\hline MOA X MOP & $14,81 \pm 8,03 \times 28,45 \pm 6,94$ & $0,0310^{*}$ \\
\hline MOD X MOE & $6,25 \pm 5,28 \times 27,81 \pm 5,09$ & 0,1823 \\
\hline MOD X MOP & $6,25 \pm 5,28 \times 28,45 \pm 6,94$ & 0,1394 \\
\hline MOE X MOP & $27,81 \pm 5,09 \times 28,45 \pm 6,94$ & 0,8927 \\
\hline
\end{tabular}

(*) $\mathrm{p}<0,05$; MOA - maior oscilação anterior, MOD - maior oscilação a direita, MOE - maior oscilação a esquerda, MOP - maior oscilação posterior.

Quando se correlacionaram as medidas de maior oscilação (para os quatro lados) com o tempo de uso da prótese, com o peso da prótese e com a idade, nos indivíduos do grupo de amputados transtibial, observamos que apenas a medida de maior oscilação anterior e a idade se correlacionou negativamente $(p=0,038)$. Do mesmo modo, o grupo controle também apresentou correlação negativa entre a maior oscilação posterior e a idade $(\mathrm{p}=0,036)$.

\section{DISCUSSÃO}

Neste presente estudo, buscou-se avaliar indivíduos amputados por etiologia traumática, usuários de próteses, compreendendo um período entre 1,5 e 5 anos, concordando com os relatos de Leonard e Méier ${ }^{21} \mathrm{e}$ DeLisa e Gans ${ }^{22}$, que citam o trauma como amputação não eletiva responsável por $20 \%$ das amputações de membros inferiores.

O método utilizado, a Biofotogrametria Computadorizada, mostrou-se de fácil aplicação na avaliação do equilíbrio estático, como descreveram Cardoso ${ }^{23}$, Guimarães ${ }^{24}$ e Baraúna et al. ${ }^{25}$, deixando claro que se trata de um instrumento capaz avaliar o equilíbrio quantitativamente, ou seja, com maior fidedignidade.

Analisando os resultados, observamos que os amputados transtibiais tiveram significativa oscilação para o lado esquerdo, comparado aos outros grupos, sendo importante salientar que $88,88 \%$ dos componentes deste grupo eram amputados transtibiais direito. Tais resultados podem ser facilmente compreendidos por Geurts e Mulder ${ }^{26}$, os quais em seus relatos afirmam que amputados, quando adotam posição estática tendem a ter desequilíbrios para o lado de maior massa, ou seja, o lado não amputado. Isakov et al. ${ }^{14}$ também explicam este fato; ao compararem equilíbrio e descarga de peso entre amputados transtibiais com indivíduos não amputados, verificaram aumento das oscilações para o lado não amputado. Esses autores possibilitam uma nova explicação acerca do desequilíbrio, ao afirmarem que em amputados ocorre uma perda da informação proprioceptiva da pele, tecido subcutâneo, cápsula articular, ligamentos, tendões e músculos, como resultado da amputação. 
De acordo com os dados apresentados, pode-se observar ainda que os indivíduos amputados transfemurais obtiveram significativa oscilação anterior, quando comparados ao grupo controle, assim como diferenças significativas entre as medidas de maior oscilação anterior, comparadas às medidas de maiores oscilações posterior, esquerda e direita. O que condiz com os achados de Buckley et al. ${ }^{16}$ os quais comparam o equilíbrio estático e dinâmico de amputados de membros inferiores e verificaram que a maior oscilação é conseqüente da alteração do centro de pressão no plano anteroposterior. Realçam ainda a importância do tornozelo na manutenção dos movimentos no plano sagital, sugerindo que o desequilíbrio neste plano em amputados, ocorre devido a restrita ação do pé e joelho protético no controle dos movimentos neste plano.

No presente estudo, verificou-se duas correlações negativas. A primeira foi entre maior oscilação posterior e idade no grupo controle, e a segunda entre maior oscilação anterior e idade, no grupo dos amputados transtibiais. Observou-se então que os indivíduos não amputados possuem maior domínio corporal, pois não oscilam anteriormente, e indivíduos amputados transtibiais bem adaptados à prótese, ao atingirem a idade próxima à maturidade corporal, tendem a diminuir as oscilações anteriores, assemelhando-se aos indivíduos não amputados. Isto é explicado por Borer ${ }^{27}$, o qual versa sobre a maturidade do sistema musculoesquelético, relatando que esta é atingida em torno da terceira década de vida; o que vem corroborar com estes estudos, os quais obtiveram média de idade de 33,38 anos.

A metodologia desenvolvida no presente trabalho visou verificar se as alterações de equilíbrio de amputados poderão servir de suporte nas tomadas de decisões terapêuticas no que tange ao processo de reabilitação de amputados transtibiais e transfemurais.

\section{CONCLUSÃO}

Mediante os resultados encontrados nas condições estudadas, conclui-se que, nos indivíduos amputados transtibiais, ocorre uma correlação negativa entre medidas de oscilação anterior e idade, demonstrando que à medida que o indivíduo atinge o domínio corporal menores são as oscilações anteriores. Os amputados transfemurais protetizados têm maior tendência a oscilações anteriores, indo ao encontro a outro ponto encontrado, no qual observamos que as oscilações no plano sagital em amputados são maiores quando comparados às oscilações no plano frontal.

Conclui-se também que o tempo de uso da prótese está diretamente relacionado às oscilações, pois foi verificado que quanto maior o tempo de uso, mais o desequilíbrio tende a diminuir. E, por fim, observou-se que amputados transtibiais tendem a oscilar mais para o lado contra-lateral ao membro amputado.

Agradecimentos: à PROSUP-CAPES pelas bolsas de Mestrado.

\section{REFERÊNCIAS BIBLIOGRÁFICAS}

1. Boccolini F. Reabilitação: amputados, amputações e próteses. São Paulo: Robe Livraria e Editora; 2001.

2. Carvalho JA. Amputações de membros inferiores: em busca de plena reabilitação. $2^{\mathrm{a}}$ ed., São Paulo: Manole; 2003.

3. Bilodeau S, Hébert R, Desrosiers J. Lower limb prosthesis utilization by elderly amputees. Prosthetics and Orthotics International 2000; 24(2):126-132.

4. André JM, Paysant J, Martinet N, Beis JM. Classification et mécanismes dês perceptions et illusions corporelles des amputés. Annales de Réadaptation et de Médecine Physique 2001; 44(1):13-18.

5. Lusardi MM, Nielsen CC. Orthotics and prosthetics in rehabilitation. London: Butterworth-Heinemann; 2000.

6. Calmel P, Béthoux F, Chagnon PY, Rigal F. Échelles d'evaluation fonctionnelle et amputation du memre inférieur. Annales de Réadaptation et de Médecine Physique 2001; 44(8):499-507.

7. Ramos JRJ, Demyer W. Semiotécnica da observação clínica. $7^{\mathrm{a}}$ ed. São Paulo: Sarvier; 1986.

8. Rubenstein LZ, Robbins AS, Sculman BL. Falls and instability in the elderly. Jof the Am Geriatrics Society 1988; 36(3):266-78.

9. Machado A. Neuroanatomia funcional. $2^{\mathrm{a}}$ ed.: Rio de Janeiro: Atheneu; 1993.

10. Presumido LMB, Baraúna MA, Ferreira C, Silva KC. Estudo comparativo entre o equilíbrio estático de indivíduos sedentários e não sedentários do sexo feminino. Ícone 1995; 3(2): 39-62.

11. Aruin AS, Nicholas JJ, Latash ML. Anticipatory postural adjustments during standing in below-the-knee amputees. Clinical Biomechanics 1997; 12(1):52-59.

12. Cunha RPF, Brito MMT, Prazeres EMB, Filho NTP. Plasticidade neural e a neuropatia periférica diabética. Fisioterapia Brasil 2002; 3(2):108-15.

13. Baraúna MA, Canto RST, Oliveira AS, Soares AB, Silva CDC, Cardoso FAG. Avaliação do equilíbrio estático do portador de diabetes mellitus pela biofotogrametria. Diabetes Clínica 2003; 7(1):57-62.

14. Isakov E, Mizrahi J, Ring H, Susak Z, Hailim N. Standing sway and weight gearing distribution in people with below-knee amputations. Arch Phys Med Rehabil 1992; 73:174-78.

15. Mouchnino L. Postural reorganization of weight-shifting in below-knee amputees during leg raising. Experimental Brain Research 2000; 121(2):205-214. 
16. Buckley JG, O’Driscoll D, Bennett SJ. Postural sway and active balance performance in highly active lower-limb amputees. American Journal of Physical Medicine and Rehabilitation 2002; 81(1):13-20.

17. Baraúna MA. Estudo comparativo entre a avaliação do equilíbrio estático de indivíduos amputados e não amputados [Tese Doutorado em Motricidade Humana]. Lisboa(Portugal): Universidade Técnica de Lisboa; 1997.

18. Passant U, Warkentin S, Gustafson L. Orthostatic hypotension and low blood pressure in organic dementia: a study of prevalence and related clinical characteristics. Inter J Geriatric Psychiatry 1997; 12 (3): 395-403.

19. Ricieri DV. Validação de um protocolo de fotogrametria computadorizada e quantificação angular do movimento toracoabdominal durante a ventilação tranqüila [Dissertação de Mestrado em Fisioterapia]. Uberlândia: UNITRI -Centro Universitário do Triangulo; 2000.

20. Magazoni VS. Estudo correlacional entre a expansibilidade da caixa torácica e a capacidade vital pulmonar nos indivíduos portadores e não-portadores de espondilite anquilosante [Dissertação de Mestrado em Fisioterapia]. Uberlândia: UNITRI-Centro Universitário do Triângulo; 2000.

21. Leonard JAJ, Méier R H. Próteses. São Paulo: Manole; 1992.

22. Delisa JÁ, Gans BM. Tratado de medicina física e reabilitação: princípios e prática. $3^{\mathrm{a}}$ ed. São Paulo: Manole; 2002.

23. Cardoso FAG. Avaliação do equilíbrio estático em pacientes diabéticos portadores de neuropatia autonômica, através da fotogrametria computadorizada [Dissertação de Mestrado em Fisioterapia]. Uberlândia: UNITRI-Centro Universitário do Triângulo; 1999.

24. Guimarães EA. Avaliação do equilíbrio estático de indivíduos normais através da Biofotogrametria Computadorizada e da Oscilometria [Dissertação de Mestrado em Fisioterapia]. Uberlândia: UNITRI-Centro Universitário do Triângulo; 2003.

25. Baraúna MA, Barbosa SRM, Canto RST, Silva RAV, Silva CDC, Baraúana KMP. Estudo do equilíbrio estático de idosos e sua correlação com quedas. Rev Fis Bras 2004; 5(2,):136-41.

26. Geurts ACH, Mulder TW. Attention demands in balance recovery following lower limb amputation. J Motor Behav 1994; 26:162-170.

27. Borer KT. The effects of exercise on growth. Sports Medicine 1995; 20(6):375-97. 